\title{
Influenza del calcio sulla morfologia degli eritrociti urinari
}

\author{
M. Ballestri, A. Baraldi, A. D'Amelio e D. Bonucchi \\ Cattedra e Divisione di Nefrologia, Azienda Policlinico - Università di Modena
}

$\mathbf{N}$

umerosi studi hanno suggerito fin dalla fine del secolo scorso che la morfologia degli eritrociti urinari (EU) potesse fornire utili ragguagli sulla loro provenienza (1-3). Nel 1982 gli australiani Fairley e Birch $(4,5)$ hanno introdotto nella pratica clinica lo studio degli EU con microscopio a contrasto di fase (MCF), sfruttandone la capacità di evidenziare al meglio i dettagli morfologici delle cellule non colorate in sospensione. Con questo strumento risulta semplice distinguere le alterazioni morfostrutturali degli EU dovute al passaggio trans-glomerulare, come l' acantocitosi (Fig. 1), da modificazioni indotte dai caratteri fisico-chimici, come l'echinocitosi (Fig. 2). È quindi possibile utilizzare l'esame, ormai citato in letteratura come test di Fairley, per diagnosticare l'origine glomerulare o non glomerulare di una microematuria (ME). Va inoltre sottolineato come l'impiego del MCF consenta un migliore studio del sedimento urinario nel suo complesso: basti ricordare al proposito che i cilindri jalini possono essere del tutto invisibili al microscopio ottico convenzionale a causa del loro basso indice di rifrazione (6).

\section{Meccanismi responsabili del dismorfismo degli eritrociti urinari}

La fisiopatologia del dismorfismo degli EU non è stata ancora completamente chiarita: le legittime perplessità sulla responsabilità del passaggio trans-glome- rulare nascono dal fatto che nell'ematuria $\mathrm{i}$ globuli rossi vengono a trovarsi in un ambiente non fisiologico, con osmolalità e pH assai diversi da quelli plasmatici, in presenza di diversi soluti ad elevata concentrazione. Alcune osservazioni tratte da modelli sperimentali sembrano escludere che le alterazioni morfologiche degli EU siano indotte dalle proprietà chimico-fisiche dell'urina. Il momento di raccolta del campione urinario nel corso della giornata ed il tempo intercorso fra la raccolta e l'esecuzione dell'esame morfologico non sembrano avere influenza rilevante sulla comparsa del dismorfismo eritrocitario. Il tempo di sosta del campione invece può condizionare una sottostima delle emazie presenti per fenomeni di emolisi, particolarmente frequenti nei campioni ad elevato $\mathrm{pH}$ urinario (7-9). L'osmolalità dell' urina

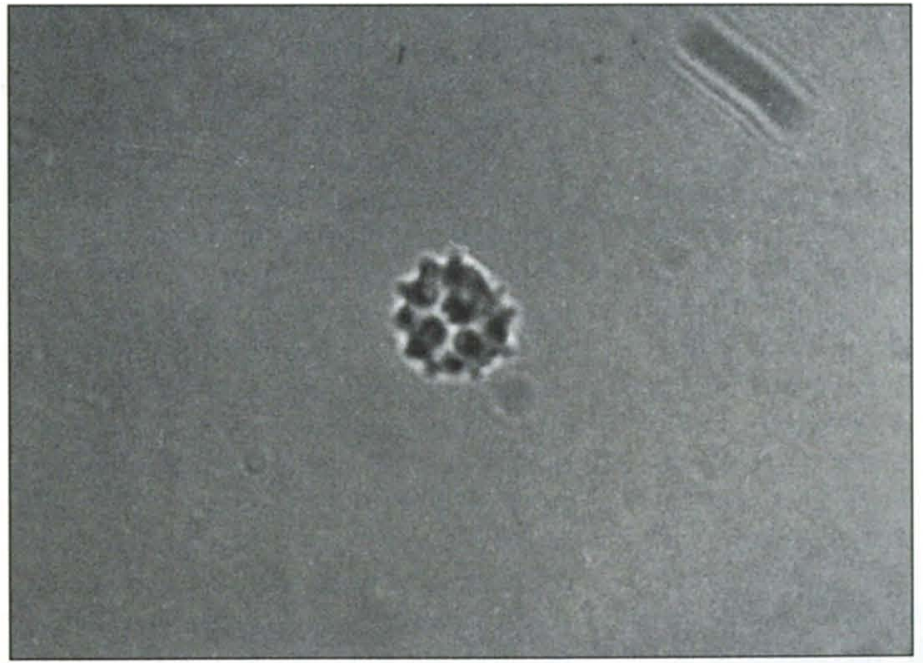

Fig. 1 - Echinocita (Microscopio a contrasto di fase $1000 x$ )

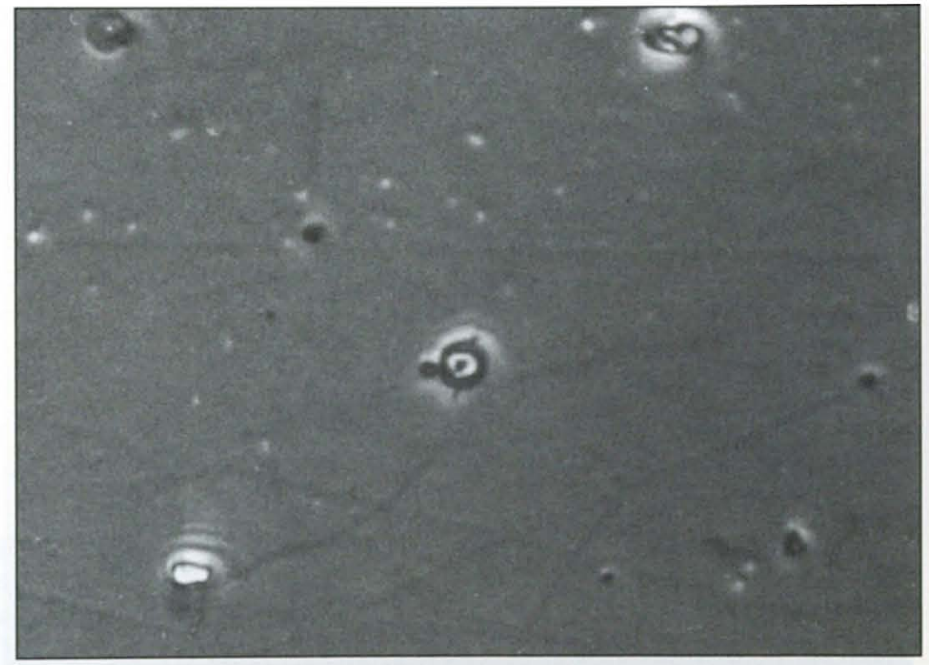

Fig. 2 - Acantocita (Microscopio a contrasto di fase 1000 x) 

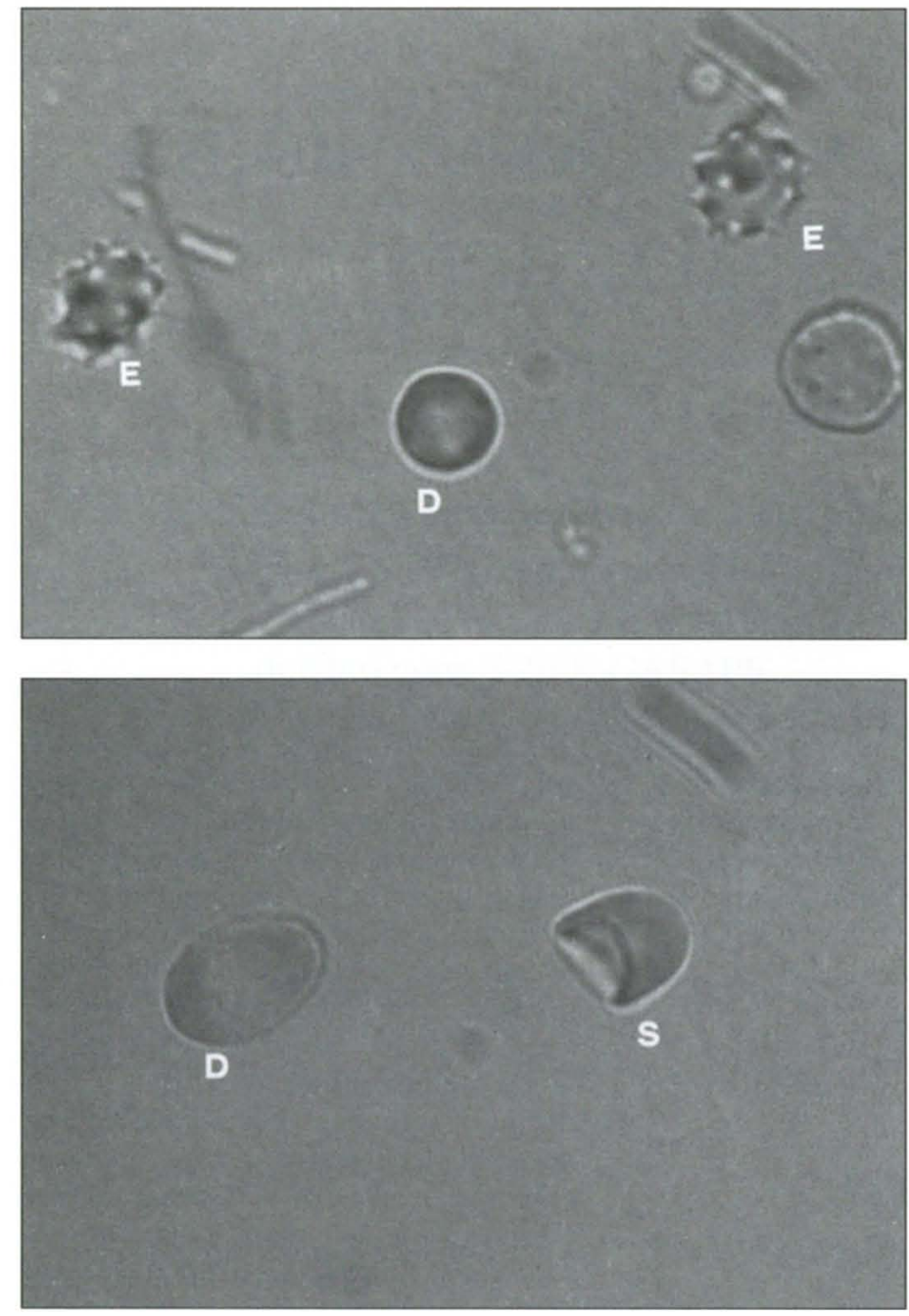

può a sua volta indurre nell' eritrocita trasformazioni di tipo discocita-echinocita (Fig. 3), discocita-sferocita (Fig. 4) oppure emolisi (10), ma non acantocitosi od altre alterazioni tipiche della ME glomerulare (11) ed inquadrabili nelle classi $\mathrm{G}$ identificate dalla recente classificazione morfologica proposta da Tomita (12). In questi casi il dismorfismo sarebbe la conseguenza del danno meccanico causato dal transito dei globuli rossi attraverso i "gaps" dei capillari glomerulari, come suggeriscono gli studi di microscopia elettronica (13) e la riproducibilità delle alterazioni ottenute ultrafiltrando emazie attraverso pori con diametro di 0.3-5 mm $(8,14)$. Opinioni alternative attribuiscono il danno eritrocitario ad enzimi presenti nell' urina, all'azione di cellule fagocitarie od a fattori rilasciati dalle stesse emazie $(15,16)$, così come potrebbero avere un ruolo chiave fattori immunologici umorali e/o cellulari (17). È comunque probabile che tutti i mecca-
Fig. 3 - Trasformazione discocita $(D)$-echinocita (E) (Microscopio a contrasto di fase $1000 x$ )

Fig. 4 - Trasformazione discocita (D)-sferocita (S) (Microscopio a contrasto di fase $1000 x$ )

nismi fisico-chimici e biologici descritti intervengano a diversi livelli sovvertendo irreversibilmente lo scheletro di membrana (SM) dell' eritrocita, la cui integrità garantisce il mantenimento della forma cellulare. Le alterazioni morfologiche "non glomerulari", come ad esempio l' echinocitosi, non dipendono da lesioni del citoscheletro e pertanto sono entro certi limiti reversibili (18), viceversa le emazie dismorfiche osservate nei pazienti affetti da patologie glomerulari, presentano perdite significative di spectrina e proteina della banda 3 (19), che sono elementi costitutivi dello SM. Nella nostra esperienza anche elevate concentrazioni urinarie di calcio $(\mathrm{CaU})$ possono indurre la comparsa di dismorfismi a carico degli EU, così da configurare quadri morfologici di ME mista o addirittura glomerulare (20). Questa possibilità non dovrebbe quindi essere trascurata e l'esclusione dell' ipercalciuria dovrebbe rientrare nell' algoritmo diagno- stico di tutte le microematurie isolate, senza cilindruria e/o leucocituria significative, anche se di origine glomerulare al test di Fairley (21).

\section{Ruolo del calcio}

È ormai noto che l'ipercalciuria, definita come escrezione urinaria di Calcio $>4$ $\mathrm{mg} / \mathrm{kg} /$ die, può associarsi ad ematuria macro o microscopica, anche in assenza di nefrolitiasi e la sua frequenza nei pazienti con ematuria isolata asintomatica varia dal 2.9 al $35 \%$ a seconda delle casistiche (22-32).

La patogenesi dell'ematuria nei pazienti ipercalciurici non è nota e di difficile dimostrazione sono le ipotesi fino ad ora formulate. Il sanguinamento potrebbe essere attribuito ad un processo flogistico interstiziale, allo sviluppo di aree di nefrocalcinosi, a lesioni focali tubulari od a lesioni epiteliali da parte di microcalcoli $(23,25,32,34)$; si tratterebbe comunque di alterazioni a carico di strutture extraglomerulari e/o delle vie escretrici, in accordo con il reperto di EU eumorfici, più volte segnalato da studi in contrasto di fase $(14,25,28,30-32,35)$. In letteratura tuttavia è possibile identificare casi in cui l' elevata escrezione di calcio si associa alla presenza nelle urine di emazie dismorfiche, in quantità significativa. (11, 12, 14, 36-39).

Poichè anche nella nostra casistica, confermata da una precedente osservazione (39), un'elevata $\mathrm{CaU}$ è presente nel $12 \%$ dei pazienti affetti da ME dismorfica isolata asintomatica, abbiamo rivolto particolare attenzione ai possibili effetti di questo elemento sulla morfologia degli EU.

Desiderando verificare gli effetti di elevate $\mathrm{CaU}$ sugli $\mathrm{EU}$, abbiamo utilizzato un modello sperimentale (20), illustrato nella (Fig. 5), in cui aliquote di $1 \mathrm{ml}$ di sangue periferico, ottenuto mediante prelievo eparinato da volontario sano a dieta libera, sono state incubate a $37^{\circ} \mathrm{C}$ in recipienti sterili contenenti $12 \mathrm{ml}$ di urina dello stesso soggetto, preventivamente studiata per escludere la presenza di microematuria e/o altre anomalie del sedimento (Tab. I). Ad osmolalità e pH costanti, la $\mathrm{CaU}$ è stata portata a $5,10,20 \mathrm{e}$ $40 \mathrm{mM} / \mathrm{l}$, mediante addizione di $\mathrm{CaCl}_{2}$, prima della sospensione delle emazie.

Analogamente si è proceduto con emazie preincubate a $37^{\circ} \mathrm{C}$, per 5 minuti, con l'isomero levogiro del Verapamil (l-V) 
(Knoll Farmaceutici S.p.A.) a differenti concentrazioni: 5, 100 e $200 \mathrm{mM}$ (40), prima della sospensione nell'urina.

Dal tempo 0 a 240 minuti, ad intervalli regolari di 30 minuti, le emazie sono state osservate indipendentemente da 2 operatori con MCF (Nikon Optiphot, Nippon Kogaku K.K., Tokyo, Japan), contate e classificate secondo i criteri di Tomita (12).

Per la conta degli eritrociti è stato utilizzato il Kowa slide (ICL Scientific, Fountain Valley, Calif., U.S.A.) ed il grado di disaccordo fra i 2 operatori è risultato sempre inferiore al $10 \%$. Ancora, con il MCF sono stati controllati lo striscio periferico del donatore e, ad intervalli regolari, le emazie periferiche preincubate con $1-\mathrm{V}$, per escludere alterazioni morfologiche intrinseche oppure indotte direttamente dal farmaco. L'esperimento è stato ripetuto tre volte utilizzando diversi donatori.

Al tempo 0 le emazie presentavano un quadro omogeneo, con elementi eumorfici, uguali fra loro e regolari (Fig. 6), così come lo striscio delle emazie prima e dopo la preincubazione con $\mathrm{l}-\mathrm{V}$, non evidenziava alterazioni morfologiche, nè volumetriche.

Con il passare del tempo comparivano modificazioni morfo-volumetriche nei campioni con $\mathrm{CaU}>10 \mathrm{mM} / \mathrm{l}$; in particolare nei primi 60 minuti si osservava una progressiva microcitosi interessante oltre 1'80\% degli elementi, con disomogenea distribuzione dell'emoglobina (Fig. 7-9). Dopo 240 minuti di incubazione (Fig. 10) era possibile rilevare gradi maggiori di dismorfismo, quali interruzioni e protrusioni di membrana, assimilabili alle classi G2-G5 proposte da Tomita (12). Fra le tipologie eritrocitarie osservate si segnalava una emazia con superficie irregolare e bordi ampi, rilevati, vagamente simile ad una ruota di carro, talvolta descritta nei pazienti affetti da ipercalciuria e/o nefrolitiasi (Fig. 11) $(21,41)$.

Inaspettatamente la comparsa delle alterazioni eritrocitarie aveva maggiore latenza nel corso della terza prova, pur manifestandosi comunque entro i 240 minuti di osservazione.

Le emazie preincubate con $1-\mathrm{V} 10 \mathrm{mM}$ risultavano morfologicamente alterate in quota minore ed inversamente proporzionale alla concentrazione di calcio nel mezzo, mentre gli eritrociti preincubati con $1-\mathrm{V}>100 \mathrm{mM}$ rimanevano costante-
Fig. 5 - Modello sperimentale: emazie periferiche $(E)$ sono state sospese nell'urina dello stesso soggetto $(U)$ con concentrazione di calcio modificata mediante addizione di $\mathrm{CaCl}_{2}(5,10,20$ e 40 $\mathrm{mM})$. Analogamente si ̀̀ proceduto con emazie preventivamente incubate con $l$ Verapamil alle concentrazioni di 10, 100 e $200 \mathrm{mM}$.

\section{TABELLA I - CARATTERI CHIMICO FISICI DI BASE DELLE URINE UTILIZ- ZATE NELLE PROVE}

$\begin{array}{llcc}\mathrm{pH} & & & \\ & & & \\ \text { Osmolalità } & \mathrm{mosm} / \mathrm{kg} & 5.5 & 6.18 \\ \mathrm{Na} & \mathrm{mmol} / \mathrm{l} & 570 & 760 \\ \mathrm{~K} & \mathrm{mmol} / \mathrm{l} & 23.3 & 59.1 \\ \mathrm{Ca} & \mathrm{mmol} / \mathrm{l} & 0.08 & 2.6 \\ \mathrm{Mg} & \mathrm{mmol} / \mathrm{l} & 1.95 & 5.05 \\ \mathrm{CI} & \mathrm{mmol} / \mathrm{l} & 73 & 174 \\ \mathrm{P} & \mathrm{mmol} / \mathrm{l} & 9.04 & 18.5\end{array}$

Fig. 6 - Emazie periferiche eumorfiche, sospese nell'urina dello stesso donatore al tempo 0 ( $\mathrm{Mi}$ croscopio a contrasto di fase 400 x). Da: Bonucchi $D$ et al. Influence of urinary calcium concentration on erythrocyte morphology. Nephron, Karger, Basel 1996; 74 $661-7$.

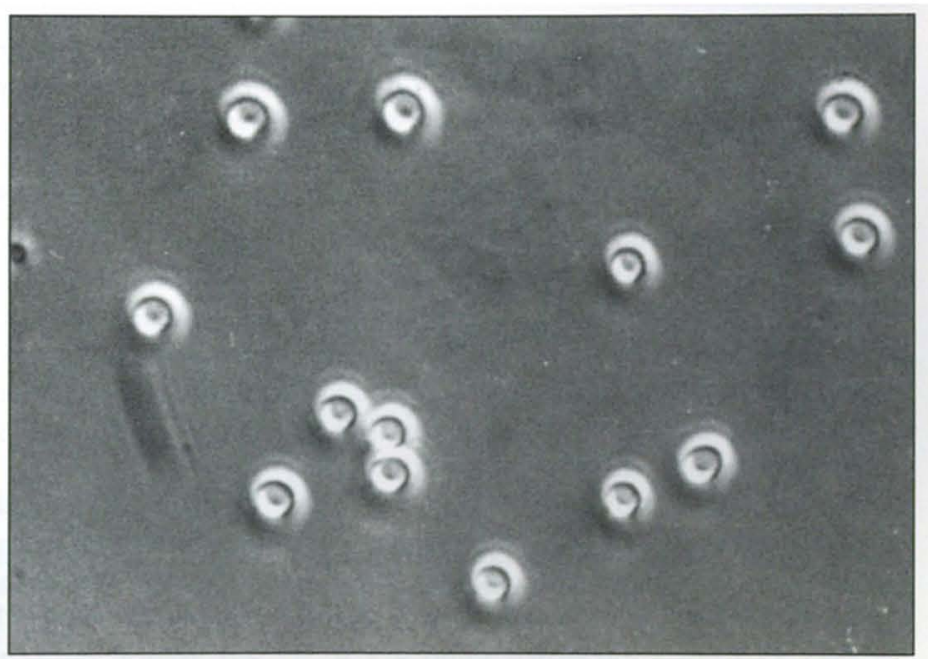




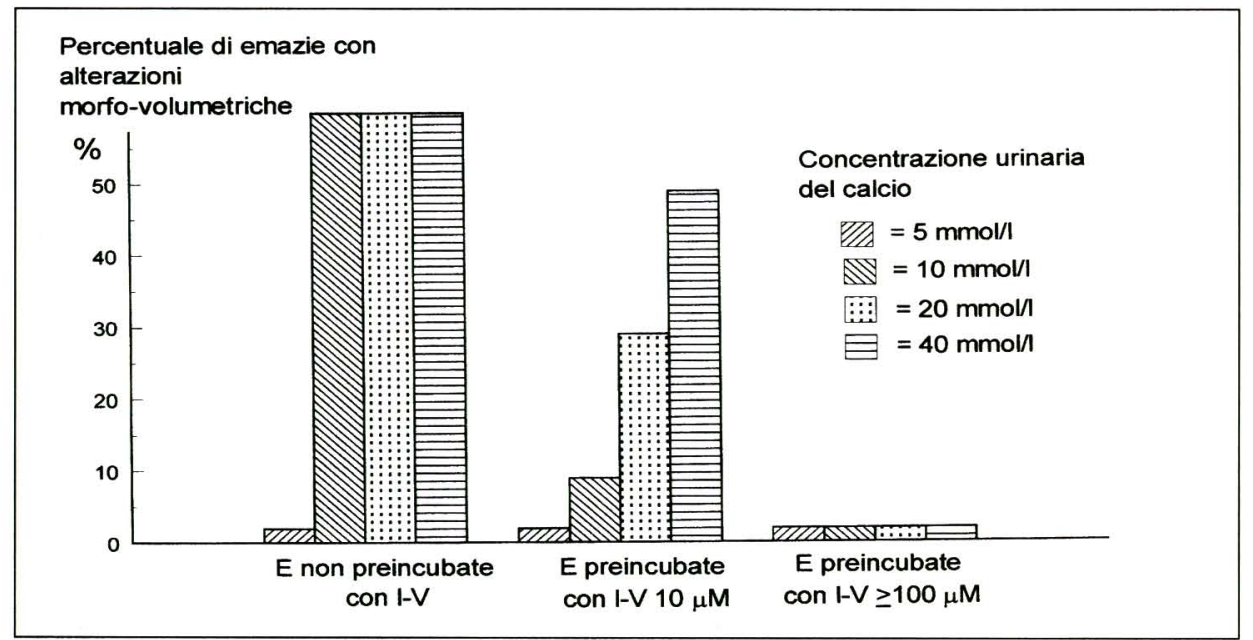

Fig. 7 - Percentuale approssimativa di emazie con alterazioni morfo-volumetriche dopo 30 minuti di sospensione nelle urine a diversa concentrazione di calcio. Le emazie preincubate con l-Verapamil $10 \mathrm{mM}$ presentavano modificazioni in quota crescente all'aumentare della concentrazione del calcio, mentre le emazie preincubate con l-Verapamil $>100 \mathrm{mM}$ risultavano inalterate.

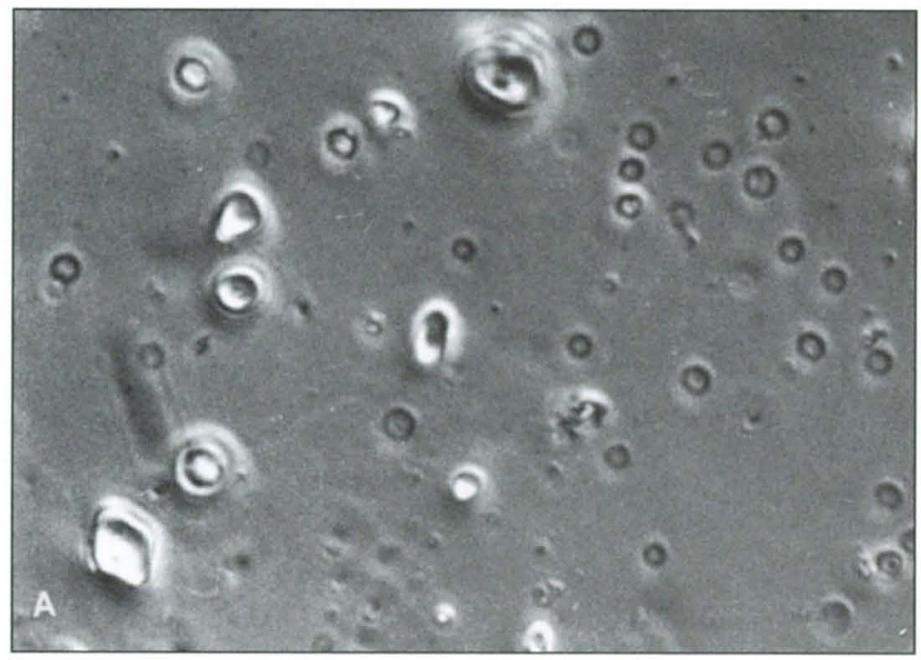

Fig. 8 - Modificazioni morfo-volumetriche delle emazie sospese in urina con concentrazione di calcio $10 \mathrm{mM}$, dopo 60' (Microscopio a contrasto di fase 400 $x$ ). Da: Bonucchi $D$ et al. Influence of urinary calcium concentration on erythrocyte morphology. Nephron, Karger, Basel 1996; 74: 661-7. mente inalterati (Fig. 12), indipendentemente dalla concentrazione del calcio. La comparsa de novo di EU microcitici e dismorfici pur in assenza di passaggio transglomerulare e la capacità del pretrattamento con $1-\mathrm{V}$ di prevenire il fenomeno, confermerebbe il ruolo chiave dello ione calcio nel processo osservato.

\section{Anatomia funzionale della membrana eritrocitaria. Influenze del calcio}

La tipica forma del globulo rosso, nonché la stabilità della membrana e le sue caratteristiche reologiche sono garantite dall'integrità dello SM. Questo risulta

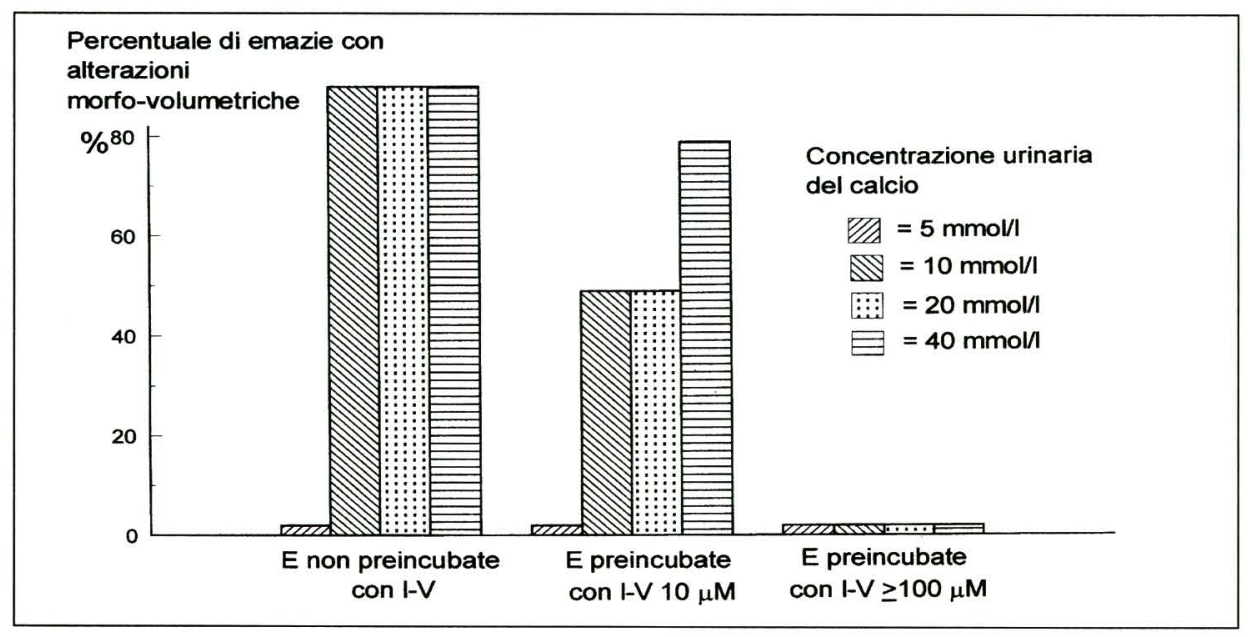

Fig. 10 - Percentuale approssimativa di emazie con alterazioni morfo-volumetriche dopo 240 minuti di sospensione nelle urine a diversa concentrazione di calcio. Le emazie preincubate con l-Verapamil $>100 \mathrm{mM}$ non presentavano modificazioni. 


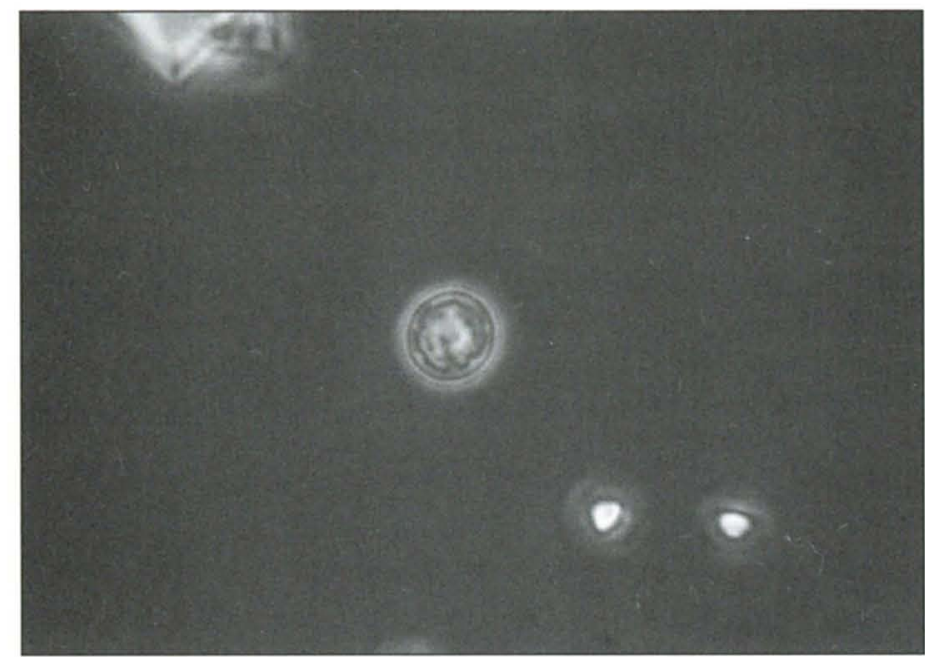

Fig. 11 - Emazia con superficie irregolare, bordi ampi rilevati; queste caratteristiche morfologiche sono talora osservabili nei pazienti con nefrolitiasi. (Microscopio a contrasto di fase $(400 x)$.

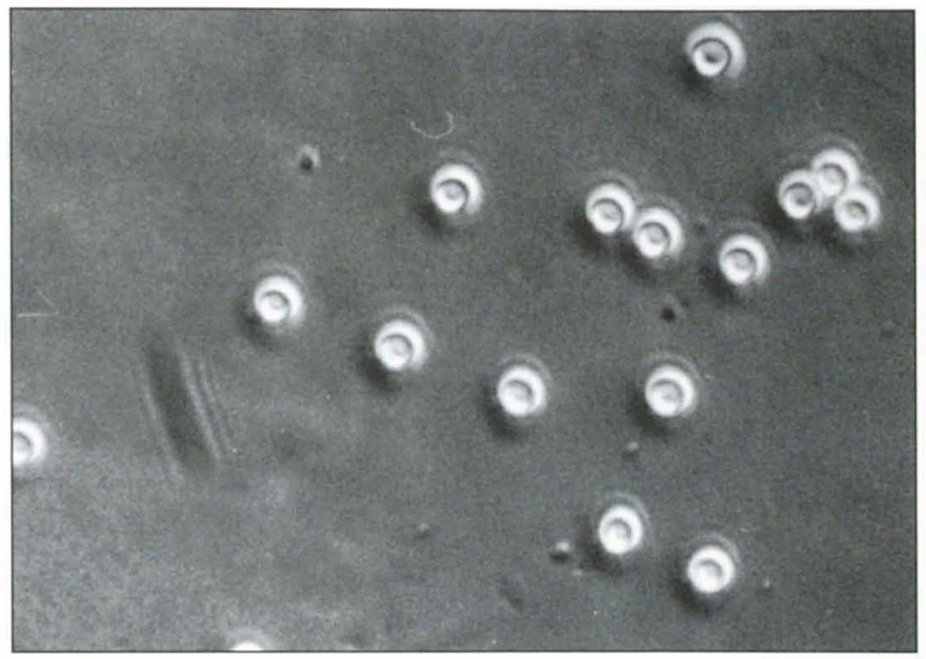

Fig. 12 - Tempo 240'. Emazie eumorfiche, preincubate con l-Verapamil $200 \mathrm{mM}$, sospese in urina con concentrazione di calcio $40 \mathrm{mM}$. (Microscopio a contrasto di fase 400 x). Da: Bonucchi D et al. Influence of urinary calcium concentration on erythrocyte morphology. Nephron, Karger, Basel 1996; 74: 661-7. costituito da una complessa rete proteica che si sviluppa sul versante citoplasmatico della membrana e si ancora ad essa tramite molecole specializzate (Fig. 13) (42, 43).

Principale costituente dello SM è la spectrina (banda 1 e 2 all'esame elettroforetico su poliacrilammide con sodio dodecilsolfato) (44), eterodimero costituito da due catene, a e b, di 240 e 220 KD rispettivamente. Le molecole di spectrina sono unite fra loro nella sequenza testa-coda mediante complessi di giunzione (CG) costituiti da F-actina (banda 5) e proteina della banda 4.1. La stabilizzazione del legame spectrina-actina è favorito dalla presenza di altre 2 frazioni proteiche: l'adducina, in duplice forma di 97 e $103 \mathrm{KD}$, e la demantina (banda 4.9) che sembra partecipare all'organizzazione dei filamenti di actina. Discussa rimane la precisa localizzazione di altri costituenti identificati quali tropomiosina, miosina, tropomodulina e caldesmon $(42,45)$.

Questo network proteico si estende per $1100 \mathrm{~mm}^{2}$ sotto la faccia citoplasmatica della membrana (45) e si ancora ad essa tramite il legame fra la banda 4.1 e la glicoforina, proteina eritrocitaria di transmembrana. All' ancoraggio partecipa pure l'ancorina (banda 2.1 e 2.2), proteina globulare di $210 \mathrm{KD}$ appartenente alla famiglia della sindeine, che tramite due domini specializzati lega da un lato la spectrina e dall'altro la regione N-terminale della proteina della banda 3. Quest'ultima è la proteina di transmembrana

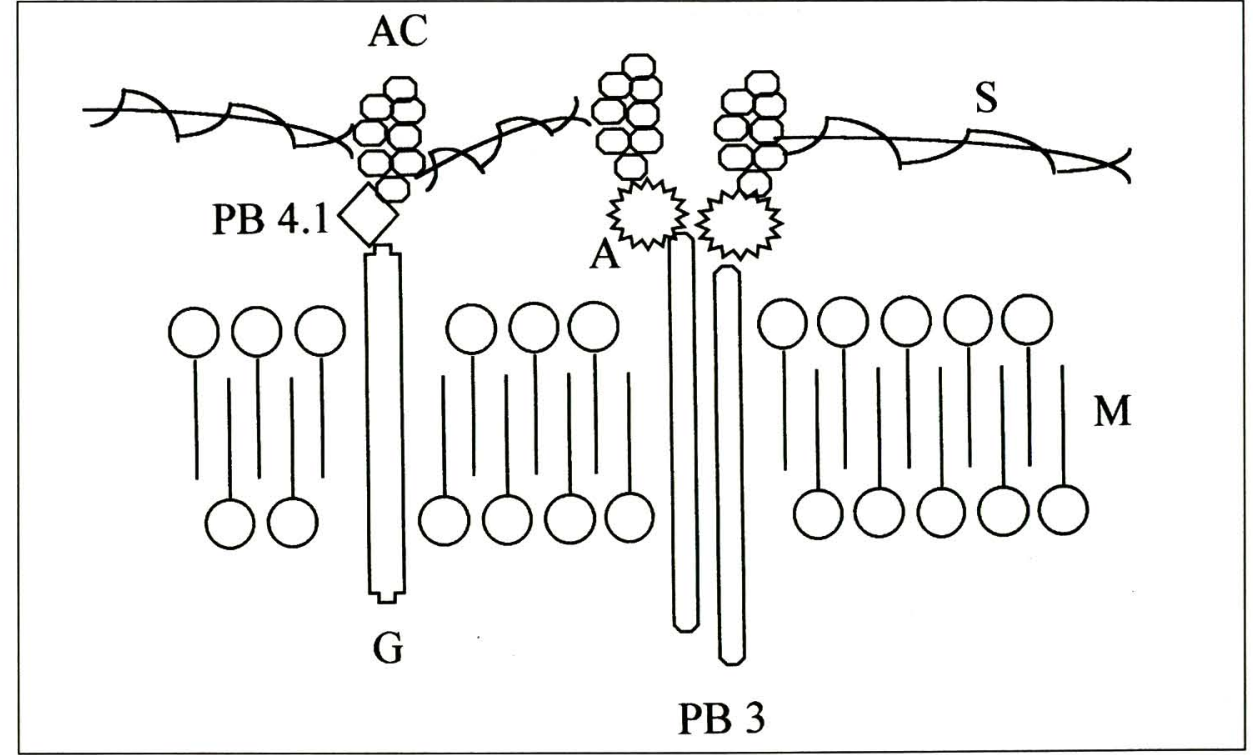

Fig. 13 - Rappresentazione schematica dello scheletro di membrana del globulo rosso; $M=$ membrana plasmatica; $G=$ glicoforina; $P B 3=$ proteina della banda $3 ; P B 4.1=$ proteina della banda $4.1 ; A=$ ancorina; $A C=$ actina; $S=$ spectrina. Le molecole di $S$ sono unite fra loro in sequenza testa-coda a livello dei complessi di giunzione formati da $A C$ e PB 4.1.

maggiormente rappresentata nell'emazia $\mathrm{e}$, mentre la porzione intramembranosa catalizza lo scambio $\mathrm{Cl} / \mathrm{HCO}_{3}$, la catena citoplasmatica comprende siti di legame per l' ancorina, le proteine della banda 4.1 , della banda 4.2 e gli enzimi della glicolisi $(42,45)$.

L'integrità dello scheletro di membrana è indispensabile per il mantenimento della forma, nonché per conferire all'emazia la caratteristica elasticità, che consente uno stiramento unidirezionale re- versibile fino a $24 \mathrm{~mm}$ (pari a circa 2.8 volte il suo diametro) $(45,46)$.

Il calcio può interagire a diversi livelli con i costituenti dello SM (Fig. 14); 1'aumento della sua concentrazione citoplasmatica infatti è in grado di attivare la proteinkinasi $\mathrm{C}$ (PKC), responsabile a sua volta della fosforilazione della proteina della banda 4.1, nonché della demantina e dell'adducina con conseguente inibizione del legame spectrina-banda 4.1 e della formazione del CG. Già in- 


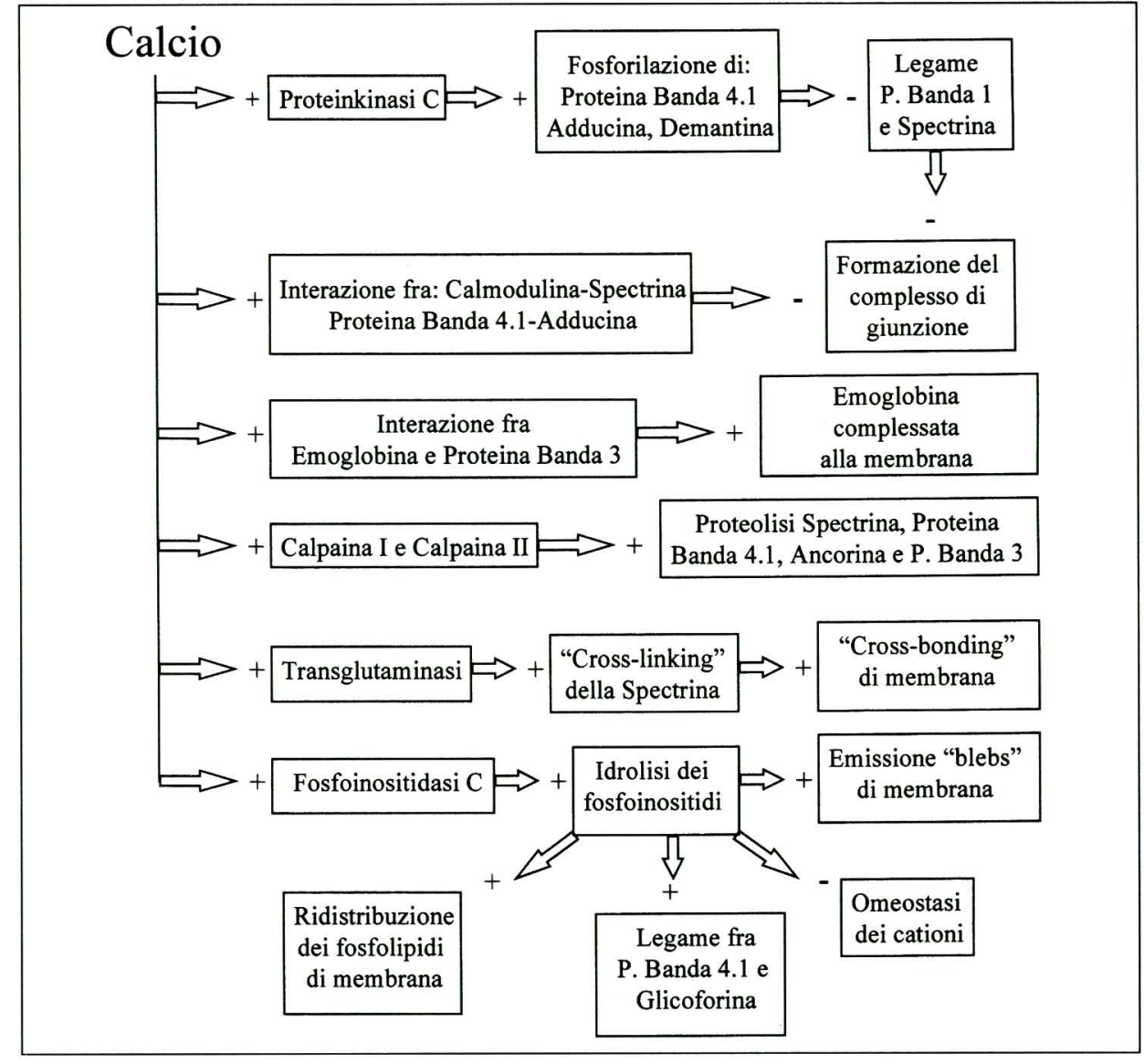

Fig. 14 - Interazioni fra il calcio e lo scheletro di membrana dell'eritrocita. Il segno + indica un effetto di stimolo, il segno - indica invece l'effetto inibitorio elo sfavorevole.

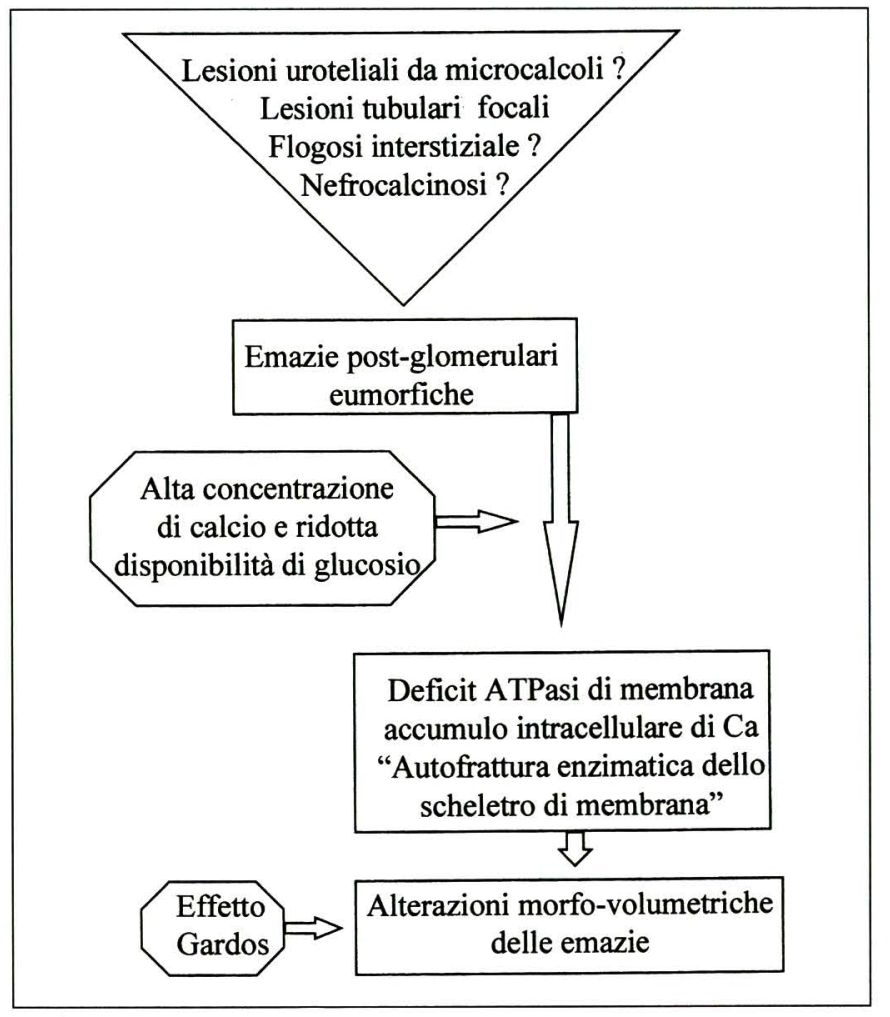

Fig. 15 - Possibile spiegazione della presenza di emazie dismorfiche nelle urine dei pazienti affetti da ipercalciuria con o senza nefrolitiasi. crementi micromolari del calcio intracellulare sembrano influenzare direttamente, nell'eritrocita, l'interazione fra calmodulina e molecole di spectrina, banda 4.1 ed adducina, con compromissione della formazione del CG $(42,43,45,47)$. Le calpaine I (m-calpain) e II (m-calpain) sono attivate in vitro dal calcio a concentrazioni rispettivamente micro e millimolari ed hanno attività enzimatica proteolitica nei confronti della spectrina, della banda 3, ma soprattutto della banda 4.1 e della ancorina. L'effetto di questi enzimi sembra avere profonde conseguenze sulle proprietà dell'emazia e sulla sua morfologia, fino alla frammentazione $(42,47)$.

È d'altronde nota la capacità del calcio di promuovere l'idrolisi enzimatica del fosfatidilinositolo-4-5-difosfato e 4-fosfato da parte della fosfolipasi $\mathrm{C}$, con incremento dell' 1,2 diacilglicerolo e del fosfatidato (48-50). Tale evento si ripercuote negativamente sull'omeostasi intracellulare dei cationi poiché riduce l'effetto chelante esercitato dai gruppi fosfato dell'inositolo e modifica l'attività dell'ATPasi Mg dipendente. Questa provvede al mantenimento della concentrazione intracellulare del calcio ionizzato entro i normali limiti di $20-40 \mathrm{nmol} / \mathrm{L}$ (42). La riduzione del contenuto in fosfoinositidi condiziona inoltre una ridistribuzione dei fosfolipidi di membrana $(42,48)$, processo che sembra essere responsabile del blebbing di membrana calcio indotto e che potrebbe avere parte anche nella crenazione da deplezione di $\operatorname{ATP}(50,51)$. Ancora, viene inibito il legame della banda 4.1 con la glicoforina, interazione che, in vitro, dipende strettamente dalla presenza di fosfatidilinositolo-4.5-difosfato (42).

Altri dati sperimentali suggeriscono come i livelli di calcio intracitoplasmatico possano influenzare l'interazione fra emoglobina e proteina della banda 3 , modulando la quota di emoglobina complessata alla membrana con conseguenti modificazioni morfo-reologiche (52).

Il sovraccarico di calcio del mazzo intracellulare favorisce l'attività transglutaminasica con "cross-linking" delle proteine di membrana e conseguente "crossbonding" della membrana stessa; tale processo porta all' adesione fra aree opposte della faccia citoplasmatica della membrana dell'eritrocita e potrebbe essere correlato ai fenomeni di blebbing. $(52,53)$. 
Si può pertanto ipotizzare che emazie similperiferiche provenienti da lesioni extraglomerulari correlate all'ipercalciuria, in un microambiente ricco di calcio e povero di substrati energetici tali da garantire il corretto funzionamento delle pompe di membrana, accumulino progressivamente calcio con conseguente danno strutturale del citoscheletro associato poi a modificazioni morfo-volumetriche (Fig. 15). La microcitosi osservata nel nostro modello sperimentale potrebbe anche essere spiegata, almeno in parte, dall'efflusso di potassio dall'emazia per effetto Gardos: l'ingresso di calcio nel globulo rosso induce infatti una fuoriuscita di potassio ed acqua con decremento del volume globulare $(50,54,55)$. L'incostanza temporale rilevata nella comparsa delle modificazioni eritrocitarie, potrebbe invece essere legata a differenti velocità dei flussi ionici transmembrana realizzatisi nelle diverse prove. È Infatti noto che, in assenza di sostanze ad attività ionofora, l'ingresso del calcio nel globulo rosso è assai lento (17-170 nM min-1 1-1 red blood cells) ed è influenzato notevolmente dalla concentrazione degli altri cationi nel mezzo. In particolare, la diminuzione del gradiente del $\mathrm{Na}$ fra intra ed extracellula accelera l'ingresso del calcio, mentre lo rallenta l'abbattimento del gradiente del $\mathrm{K}+(40$, 56). Le differenze di composizione ionica delle urine utilizzate potrebbero quindi rendere ragione della ritardata comparsa di dismorfismi nel terzo esperimento. Disponiamo comunque di dati insufficienti per trarre conclusioni e non devono essere sottovalutati il contributo degli altri elementi, ionici e non, all'equilibrio elettrochimico di un sistema estremamente complesso quale è l'urina. La velocità con cui il calcio si accumula nell'eritrocita è infatti condizionata da numerosi altri fattori che includono la disponibilità di substrati energetici per la glicolisi, indispensabile al corretto funzionamento della ATPasi Mg dipendente deputata all'estrusione del calcio dalla cellula (57), e l'età del globulo rosso stesso (58).

\section{Conclusioni}

Numerosi dati suggeriscono che in corso di ematuria la concentrazione del calcio nelle urine, può influenzare la morfologia degli EU, configurando all'esame microscopico in contrasto di fase un pattern di tipo glomerulare o misto. La presenza nelle urine di emazie dismorfiche, non accompagnate da cilindruria e/o leucocituria significative, non sembra quindi sufficiente ad escludere l'ipotesi diagnostica di ipercalciuria; ne consegue che anche l'algoritmo diagnostico della ME glomerulare isolata asintomatica dovrebbe includere fra le indagini suggerite la misurazione dell'escrezione urinaria di calcio.

Qualora i nostri nostri dati vengano confermati da altri-studi, potrebbe risultare utile una revisione della nomenclatura, poiché le definizioni di più corrente uso (glomerulare/non glomerulare, glomerulare/post-glomerulare, renale/non-renale, etc.) possono risultare di non univoca interpretazione. A conferma del ruolo discriminante degli acantociti $(11,59,60)$, una classificazione più articolata, ma di maggior praticità clinica (61) potrebbe identificare pazienti con:

a) ME glomerulare con eritrociti dismorfici, acantociti in quota variabile e segni di attività del sedimento, da sottoporre ad un iter diagnostico strettamente nefrologico; b) ME dismorfica con eritrociti dismorfici, senza acantocitosi, con o senza cristalluria, da indirizzare ad iter diagnostico nefrologico comprensivo di misurazione urinaria dei metaboliti litogeni;

c) ME post-glomerulare (o similperiferica) con eritrociti integri e/o stomatociti e/o echinociti e/o altri elementi compresi nelle classi $\mathrm{N}$ di Tomita, da avviare verso indagini urologiche;

d) ME mista con combinazione in diversa proporzione degli elementi sopra descritti. In quest'ultimo caso il quadro clinico e l'età del paziente suggeriranno la priorità nefrologica od urologica dei successivi accertamenti.

\section{BIBLIOGRAFIA}

1.

Rieder H. Atlas der klinischken Mikroscopie des Harnes. Leipzig, Vogel 1898.

2. Eichorst E. Trattato di patologia e terapia speciale medica; Augusto Murri ed. S.E.L. III $^{\circ}$ edizione italiana, Milano 1906; 766-74: 8067.

3. Burgsch T, Schittenhelm A, Galli G. Metodi di ricerca clinica. U.T.E.T., Torino 1916; 468-9.

4. Birch DF, Fairley KF. A simple method for identifyng glomerular bleeding. Kidney Int 1982; 21: 105.

5. Birch DF, Fairley KF, Witworth JA et al. Urinary erythrocyte morphology in the diagnosis of glomerular hematuria. Clin Nephrol 1983; 20: 78-84.

6. Fogazzi GB, Passerini P, Ponticelli C, Ritz E. The urinary sediment. Masson, Milano 1993; 51.

7. Fogazzi GB, Passerini P, Dell'Orto A. Sedimento urinario e strisce reattive nella diagnosi di ematuria. In: Syllabus di Nefrologia Le Ematurie, Fogazzi GB, Imbasciati E, Ponticelli C ed. Wichtig Editore, Milano 1992; 25-33.

8. Briner VA, Reinhart WH. In vitro production of glomerular red cells: role of $\mathrm{pH}$ and Osmolality. Nephron 1990; 56: 13-8.

9. Rizzoni G, Braggion F, Zacchello G. Evaluation of glomerular and nonglomerular hematuria by phase-contrast microscopy. J Pediatr 1983; 103: 370-4.

10. Bessis M. Living blood cells and their ultrastructure. Translated by Weed RI, Springer Verlag, Berlin, Heildelberg, New York 1973; 146-64.

11. Kohler H, Wandel E, Brunck B. Acanthocyturia-A characteristic marker for glomerular bleeding. 
Kidney Int 1991; 40: 115-20.

12. Tomita M, Kitamoto $\mathrm{Y}$, Nakayama M, Sato T. A new morphological classification of urinary erythrocytes for differential diagnosis of glomerular hematuria. Clin Nephrol 1992; 37: 84-9.

13. Trung LJ, Hiroyoshi W, Hiroshi M, et al. Mechanism of hematuria in glomerular disease. Nephron 1983; 35: 68-72.

14. Kubota H, Yamabe H, Ozawa $K$, et al. Mechanism of urinary erythrocyte deformity in patients with glomerular disease. Nephron 1988; 48: 338-9.

15. Schramek P, Moritsch A, Haschkowitz H, Bernd R, Binder R, M. In vitro generation of dysmorphic erythrocytes. Kidney Int 1989; 36: $72-7$.

16. Roth St, Renner E, Rathert P. Diagnostik der glomerularen mikrohamaturie. Urologe 1991; 30: 127-33.

17. Di Paolo N, Garosi G, Batignani $\mathrm{T}$, et al. Dismorfismo eritrocitario urinario. Danno fisico od immunologico? Giorn It Nefrol 1991; 8 (S1): 99.

18. Bull BS, Brailsford D. Red blood cell shape. In Agre P, Parker JC (ed): Red blood cell membranes: structure, function, clinical implications. Dekker, New York: 1989: 401-21.

19. Funfstruck R, Halbhuber KJ, Baumann E, Klinger M, Ohering H, Stein G. Experimental investigations into the formation of dysmorphic erythrocytes in glomerulonephritis. Nephrol Dial Transplant 1993; 8: 919.

20. Bonucchi D, Ballestri M, Bettelli $\mathrm{F}$, et al. Influence of urinary calcium concentration on erythrocyte morphology. Nephron 1996; 74: 661-7.

21. Onucchi D, Ballestri M, Bettelli F. Does any microscopic haematuria require urinary calcium determination? (letter). Pediatr Nephrol 1995; 9: 671.
22. Langman CB, Moore ES. Hypercalciuria in clinical pediatrics. Clin Ped 1984; 23: 135-7.

23. Roy III S, Stapleton FB, Noe HN, Jerkins G. Hematuria preceding renal calculus formation in children with hypercalciuria. J Pediatr 1981; 99: 712-5.

24. Kalia A, Travis LB, Brouhard $\mathrm{BH}$. The association of idiopatic hypercalciuria and asymptomatic gross hematuria in children. J Pediatr 1981; 99: 716-9.

25. Stapleton FB, Roy III S, Noe HN, Jerkins G. Hypercalciuria in children with hematuria. N Engl J Med 1984; 310: 1345-8.

26. Stapleton FB. Idiopathic hypercalciuria: association with isolated hematuria and risk for urolithiasis in children. Kidney Int 1990; 37: 807-11.

27. Cattini Perrone $\mathrm{H}$, Ajzen $\mathrm{H}$, Toporovski J, Schor N. Metabolic disturbance as a cause of recurrent hematuria in children. Kidney Int 1991; 39: 707-10.

28. Voghenzi A, Bezzi TM, Lusardi P, Soriani S. Acquired hyperoxaluria and haematuria in children. Pediatr Nephrol 1992; 6: 356-7.

29. Moore ES, Coe FL, McMann B, Favus MJ. Idiopathic hypercalciuria in children: prevalence and metabolic characteristics. J Pediatr 1978; 92: 905-10.

30. De Santo N, Nuzzi F, Capodicasa $\mathrm{G}$, et al. Phase contrast microscopy of the urine sediment for the diagnosis of glomerular and non glomerular bleeding-data in children and adults with normal creatinine clearance. Nephron 1987; 45: 35-9.

31. Verde LL, Mancheno CR, Bayle MS, de Becerra JO. Utilidad del microscopio de contraste de fase en el diagnostico de la hematuria en pacientes pediatricos. Rev Clin Esp 1988; 182: 403-6.

32. Stapleton FB. Hematuria associated with hypercalciuria and hyperuricosuria: a practical approach. Pediatr Nephrol 1994; 8: 756-1.
33. Alconcher LF, Castro C, Quintana D et al. Urinary calcium excretion in healthy school children. Pediatr Nephrol 1997; 11: 186-8.

34. Hufnagle KG, Khan SN, Penn D, Cacciarelli A, Williams P. Renal calcifications: a complication of long-term furosemide therapy in preterm infants. Pediatrics 1982; 70 : 360-3.

35. Stapleton FB. Morphology of urinary red blood cells: a simple guide in localizing the site of hematuria. Pediatr Clin North Am 1989; 34: 561-71.

36. Schramek P, Schuster FX, Georgopoulos M, Porpaczy P, Maier M. Value of urinary erythrocyte morphology in assessment of symptomless microhaematuria. Lancet 1989; ii: 1316-9.

37. Raman GV, Pead L, Lee HA, Maskell R. A blind controlled trial of phase-contrast microscopy by two observers for evaluating the source of haematuria. Nephron 1986; 44: 304-8.

38. Fassett RG, Horgan BA, Mathew TH. Detection of glomerular bleeding by phase-contrast microscopy. Lancet 1982; i: 1432-4.

39. Vaisbich MH, Kirsztajn GM, Sesso R, Pereira AB. Ajzen H. Metabolic study in dismorphic hematuria. Nephron 1992; 60: 127.

40. Varecka L, Carafoli E. Vanadate-induced movements of $\mathrm{Ca}^{2+}$ and $\mathrm{K}^{+}$in human red blood cells. J Biol Chem 1982; 257: 7414-21.

41. Birch DF, Fairley KF, Becker GJ, Kinkaid-Smith P. A color atlas of urine microscopy. Chapman \& Hall Medical, London, 1994; 31.

42. Choen CM, Gascard P. Regulation and post-translational modification of erythrocyte membrane and membrane-skeletal proteins. Semin Hematol 1992; 29: 244-92.

43. Liu SC, Derick LH, Palek J. Dependence of permanent deformation of red blood cell membranes on spectrin dimer-tetramer equilibrium: implication for permanent membra- 
ne deformation of irreversibly sickled cells. Blood 1993; 81: 522-8.

44. Rawn JD. Biochimica. Milano, Mc Graw Hill Italia, 1990; 228-32.

45. Liu SC, Derick LH. Molecular anatomy of the red blood cell membrane skeleton: structure-function relationships. Semin Hematol 1992; 29: 231-43.

46. Shen BW. Ultrastructure and function of membrane skeleton; in: Agre P, Parker JC, ed. Red blood cell membranes: structure, function, clinical implications. Dekker, New York: 1989: 261-97.

47. Whatmore J, Tang EKY, Hickman JA. Cytoskeletal proteolisis during calcium-induced morphological transition of human erythrocytes. Exp Cell Res 1992; 200: 316-25.

48. Williamson P, Kulick A, Zachowski A, Schlegel RA, Devaux $\mathrm{PF}$. $\mathrm{Ca}^{2+}$ induces transbilayer redistribution of all major phospholipids in human erythrocytes. Biochemistry 1992; 31: 6355-60.

49. Gascard P, Pawelczyk T, Lowenstein JM, Cohen CM. The role of inositol phospholipids in the association of band 4.1 with the human erythrocyte membrane. Eur J Biochem 1993; 211: 671-68.

50. Whitlow M, Iida K, Marshall P, Silber R, Nussenzweig V. Cells lacking glycan phosphatidylinositol-linked proteins have impaired ability to vesciculate. Blood 1993; 2: 510-6.

51. Bevers EM, Wiedmer T, Comfurius P, Shattil SJ, Weiss HJ, Zwaal RFA, Sims PJ. Defective $\mathrm{Ca} 2+$-induced microvesciculation and deficient expression of procoagulant activity in erythrocytes from a patient with a bleeding disorder: a study of the red blood cells of Scott syndrome. Blood 1992; 79: 380-8.

52. Friederichs E, Farley RA, Meiselman HJ. Influence of Calcium Permeabilization and membrane-attached hemoglobin on erythrocyte deformability. Am J Hem 1992; 41: 170-7.
53. Fisher TM. Role of spectrin in cross bonding of the red cell membrane. Blood Cells 1988; 13: 37794.

54. Gardos G. The function of calcium in the potassium permeability of human erythrocytes. Biochim Biophys Acta 1958; 30: 653.

55. Plishker GA, Chevalier D, Seinsoth L, Moore RB. Calcium-activated potassium transport and high molecuar weight forms of calpromotin. J Biol Chem 1992; 30: 2183943.

56. Parker JC, Dunham PB. Passive cation transport. In: Agre P, Parker JC, ed. Red blood cell membranes: structure, function, clinical implications. Dekker, New York: 1989: 507-61.

57. Vincenzi FK. The plasma membrane calcium pump; Red blood cell. In: Agre P, Parker JC, ed. Membranes: structure, function, clinical implications. Dekker, New York: 1989: 481-505.

58. Shiga T, Sekiya M, Maeda N, Kon K, Okazaki M. Cell age-dependent changes in deformability and calcium accumulation of human erythrocytes. Biochim Biophys Acta 1985; 814: 289-99.

59. Kytamoto Y, Tomita M, Akamine $\mathbf{M}$, et al. Differentiation of hematuria using a uniquely shaped red cell. Nephron 1993; 64: 32-6.

60. Fogazzi GB, Ponticelli C. Microscopic hematuria diagnosis and management. Nephron 1996; 72 : 125-34.

61. Bonucchi D, Ballestri M. Urinary erythrocytes. Morphology in haematuria of nephrological and urological origin. Nephrol Dial Transplantat 1994; 6: 737-8. 